de que no hay ninguna razón para suponer que en escala local el lenguaje culto es más parecido al académico que el lenguaje vulgar. En general es así, pero no necesariamente, y cuando sucede lo contrario, se crea un conflicto. Observamos, por ejemplo, que en el dialecto ultraserrano del Uruguay, tanto el lenguaje vulgar como el culto usan tí tienes, mientras que la norma nacional es tú tenés o, con una rotulación sintomática un poco más baja, vos tenés. Por consiguiente, aquí la norma local y la regional coinciden con el español académico, pero la norma nacional no. Ante este conflicto de normas, observamos que en la parte nordoccidental del ultraserrano vos tenés se está volviendo culto, mientras que las capas sociolingüisticamente más bajas siguen usando tú tienes. Esto significa que la norma nacional, pero no la académica, ha modificado la norma local, pero dejó intacta la regional. Hemos podido detectar conflictos de este tipo en diferentes partes de la América española, y creemos que una de las tareas inminentes e importantes de la investigación sociolingüística y sociodialectológica hispanoamericana debería ser la investigación detallada de las relaciones entre niveles de normas, en lugar de limitarse a la mera constatación de diferencias de formas. Consideramos que esto nos daría una mejor visión de la dinámica del lenguaje en la América española.

Universidad de Ottawa.

José Pedro Rona

\title{
AFICIÓN DE LOS EXTRANJEROS AL VINO $Y$ AL JAMÓN: NOTA SOBRE EL SENTIDO DE UNA SINTESIS CERVANTINA
}

En uno de los últimos estudiós que consagró a Cervantes ${ }^{1}$, Américo Castro reunió un gran número de hechos al parecer intrascendentes y hasta entonces desatendidos que, una vez agrupados, revelaban aludir todos al prejuicio fundamental de la limpieza de sangre. Tomaba Castro como punto de partida aquel extraño salvoconducto que en dos casos llevan, en la obra cervantina, unos extranjeros que viajan por España. Dicha observación le llevaba a relacionar el capítulo LIV de la segunda parte del Quijote, donde aparecen el morisco Ricote y sus compañeros tudescos, con el episodio del bretón en el Coloquio de los perros.

Mi propósito es mostrar que tiene más interés todavía la relación establecida por A. Castro si se toma en cuenta el aprovechamiento cervantino de un dato folklórico, acompañado de una reactualización del mismo.

Existen en efecto dos tradiciones paremiológicas que presentan respectivamente: 1) extranjeros de nacionalidad mal definida, peregrinos

1 A. Castro, "Sentido histórico-literario del jamón y del tocino", en su libro Cervantes y los casticismos españoles, Madrid, 1967, pp. 13-21. 
fingidos y borrachos verdaderos. Se les atribuye una jerigonza internacional, la lingua franca, en que dominan vocablos italianos; 2) franceses aficionados al jamón.

No es arbitrario el orden en que las acabo de presentar. En primer lugar, los refranes de la primera serie son más abundantes; hay por lo menos cinco en la gran colección de Correas - seis teniendo en cuenta una variante. Luego, como pronto se echará de ver, la imagen del peregrino que proponen parece conocida de antemano por el lector de tanto haber sido vulgarizada por textos famosos que van del Quijote a la Vida de Estebanillo González. De esta misma familiaridad a priori resultit un inconveniente: el que nos hayamos acostumbrado a considerar dicha imagen como un tópico, con lo que ni siquiera se nos ocurre someterla a un juicio crítico, ni examinar si los varios autores que hacen uso de ella la emplean siempre con los mismos fines. Sin tratar aquí de dilucidar este problema y ver lo que disimula tanta monotonía aparente, me contentaré con enumerar los refranes que he localizado en el Vocabulario de Correas, gracias a las imprescindibles notas críticas de L. Combet, para dedicarles luego en breve comentario sugerido por la comparación con la imagen del peregrino extranjero, tal como aparece en Cervantes.

\section{Primera serie de refranes: el extranjero amigo del vino.}

1. Toti li mundi es deskomunikate, preter Koka et Madrigate, i otro lugate ke tiene el nonbre del buei.

Dize el vulgo esto imitando el mal rromanze de los peregrinos estranxeros, ke todos son amigos de vino, i alaban por ello a Koka i Madrigal i Toro. "Preter" kiere dezir: "fuera de...", sakando a Koka, etc."2.

2. Ele fa tré prové, ele fai dormire, ele fai peire, e la ganga rrusa.

Del buen vino de España dize esto el rromero franzés i tudesko, i estranxeros ${ }^{3}$.

3. Kátame en Iepe, kátame en Okaña, kátame en toti li diabli.

Un rromero franchote, kon su kalabaza llena, i buena la kabeza i alegre se halló en los términos de lepes i Okaña, lugares abundantes de vino; puso en medio la kalabaza, i komenzó a rretozar arrededor, diziendo: "Kátame en lepe, kátame en Okaña"; a buelta dio kon el bordón en la kalabaza, i kebróla, i entonzes kon rregaño añadió lo postrero: "kátame en toti li diabli".

4. Kuerpo fecho plus; el que mató éste, mate kada día veinte.

Palavras de rromero franchote en okasión ke le hartaron i le llenaron la kalabaza. Eso es "fecho kuerpo plus", en un entierro adonde se rreparte la karidad a pobres, i ai komida, komo se usa por partes de Kastilla.

2 Tanto este refrán como los siguientes se citan por la edición crítica de $\mathbf{L}$. ComBET, Vocabulario de refranes y frases proverbiales, Bordeaux, 1967. Respectivamente pp. $505 b, 120 b, 379 b, 450 b, 365 b$.

3 Véase el comentario de Combet, que cita la variante: El vino tiene estas tres propiedades. ke haze dormir, $i$ rreir, $i$ las kolores al rrostro salir. 
5. Bueno va el bretón, kuando se le olvida el bordón; (o) Bueno va el rromerón, kuando olvida el bordón.

Se advertirá, en primer lugar, la gran variedad formal de estos cinco refranes; variedad que el comentario de Correas reduce a un denominador común (la afición de los extranjeros por el buen vino de España) .

Otro rasgo común de estos refranes es que todos encierran cierto grado de oscuridad. En realidad, no son todos igualmente herméticos, pero sí lo suficiente para que Correas crea necesario comentar cuatro de ellos.

En los tres primeros es de gran importancia la parodia idiomática. Tenemos allí muestras de seudo lingua franca. Se pueden relacionar toti li mundi, toti li diabli con las pocas palabras italianas que Cervantes pone en boca tanto de los franco-tudescos ("-Español y tudesqui, tuto uno bon compaño; y Sancho respondía: -iBon compaño, jura Di!") como del bretón del Coloquio ("el bretón daba voces, diziendo en lenguaje adúltero y bastardo... que le volviesen sus calzas; que en ellas tenía cincuenta escuti d'oro in oro").

La confusión que se produce entre francés y tudesco en el comentario del refrán núm. 2 es la misma, exactamente, que la que hallamos en la segunda parte del Quijote. Pues a Ricote, "que se había transformado de morisco en alemán o en tudesco" le pregunta Sancho "Dime: ¿quién te ha hecho franchote?"4.

Este asimilar a todos los extranjeros, designándolos con un mismo vocablo genérico de matices peyorativos en una reacción de xenofobia casi elemental, es fenómeno bien conocido, como señaló Rodríguez Marín en sus notas al Quijote, aunque la confusión operada a propósito de peregrinos $\mathrm{y}$, como cabe suponer, en pleno siglo xvi, entre franchotes y tudescos tenga posiblemente implicaciones religiosas que no suelen figurar en las usuales designaciones globales similares. Implicaciones por lo demás explicitadas en el famoso texto cervantino en que Ricote dice por qué se encontró bien en Francia, y mejor todavía en Alemania ${ }^{5}$.

Por último, la conmutación posible entre bretón y romerón en el refrán núm. 5 me parece de mucho interés. Ahí aparecen asociados, con idéntica fama de borrachos, personajes cuya relación en la obra cervantina fue destacada por $\mathrm{A}$. Castro. Él los relacionó por tener idénticos

4 Clais. Cast., Madrid, 1956, t. 7, p. 326.

5 “... entré en Francia, y aunque allí nos hacían buen acogimiento, quise verlo todo. Pasé a Italia, y llegué a Alemania, y allí me pareció que se podia vivir con más libertad, porque sus habitadores no miran mucho en delicadezas: cada uno vive como quiere, porque en la mayor parte della se vive con libertad de conciencia". Ibid., p. 332. Cf. otro caso de asimilación franchoie = cristiano nuevo en el Entremés del gabacho, NBAE 17, p. 186:

Francés-Yo Juan Francés, ¿qué quiere para ello?

Pulgón-Y tras esto será cristiano viejo.

Francés-Mientes, picoro, yo pobre mancebo, / mas fvive Dios! que soy cristiano nuevo. / ¿Diz que viejo? Tu viejo, que yo, hermano, / veinte años ha, no más, que soy cristiano...

Pulcón-... Vete poco a poco, / franchote, que te pierdes si no callas... 
salvoconductos. También utilizan la misma jerga italianizante que los peregrinos de Correas. Triple característica (vino ${ }^{6}$, tocino, italiano) que me inclina a interpretar el personaje del bretón del Coloquio conciliando los puntos de vista de Rodríguez Marín y Bataillon. Es sabido que en un artículo sobre aspectos del comercio sevillano ${ }^{7}$ afirmó éste, en oposición a Rodríguez Marín, que nada autorizaba a tomar bretón en el sentido más amplio de extranjero. Apoyándose en documentos históricos, demostraba Bataillon que la figura del marinero bretón debió de ser familiar a los sevillanos de comienzos del siglo xvn.

Como acabo de decir, creo que se han de conciliar ambos puntos de vista. Hay en el bretón del Coloquio una indiscutible nota de color local que pasaba inadvertida antes de la erudita intervención de Bataillon. Pero -y aquí me parece certera intuición de folklorista la de Rodríguez Marín- esto no impide que, partiendo de semejantes datos auténticos, la imaginación popular vaya edificando mitos. La variante del refrán núm. 5 evidencia que sí existe, hacia 1625, un motivo para crear en la extensión del sentido de la palabra bretón, extensión que le permite alternar con romerón. De ahí que, a pesar de esa nota de color local, sea legítimo establecer una relación entre el bretón del Coloquio y otros extranjeros de la obra cervantina, como ha hecho A. Castro 8 .

En ninguno de los refranes citados aparece, en cambio, un elemento que tiene tanto más importancia cuanto que en Cervantes se repite casi como una obsesión: me refiero a la acusación contra los extranjeros de venir a buscar fortuna en España y de ocultar su riqueza bajo una aparente miseria. Bataillon ha aclarado también que, en lo que toca a bretones y mercaderes, la acusación sin duda era justificada por las maniobras fraudulentas destinadas a sacar de Sevilla dinero contante y sonante ${ }^{9}$. Es normal, por lo tanto, que el bretón del Coloquio se ade-

( No se menciona explicitamente el vino a propósito del bretón del Coloquio. Sin embargo cabe suponer que, habiendo concertado con la Colindres "cena y noche", ha bebido suficientemente para hacer plausible la acusación de la prostituta, que lo tacha de borracho: "Ella decía que el bretón estaba borracho y que debia mentir en lo del dinero". Adviértase que en uno de los refranes citados en la primera serie (núm. 5) goza el bretón de fama de borracho. De un modo más general, ésta es la de todos los franceses, y les rale unos graciosos motes; cf. en el Entremés nuevo de Juan Francés "aposento de mosquitos" (NBAE, 18, p. 707).

7 M. Batalllon, "Vendeja", HR, 27 (1959), 228-245;"Pero no se ve, ni creo que exista -fuera de una discutible interpretación de la jerga italianizante empleada por el bretón al quejarse del robo de sus escudos-, la menor prueba en apoyo de extensión abusiva del sentido".

8 No quisicra terminar con la figura del bretón sin observar que otro refrán en que éste aparece tal vez le sirvièa a Cervantes de "fuente" para idear el encuentro con la prostituta: Kontra puta $i$ bretón no basta rrazón, CoMBeT, op. cit., p. $429 a$. Cervantes se complace a menudo en dar vida a parejas estereotipadas en fórmulas paremiolóyicas. Piénsese en los efectos múltiples logrados por la asociación de Sancho con su rocin (Topado ha Sancho con su rocin). Otro ejemplo es tal vez el episodio de los rebuznos, con la sabrosa discusión sobre si rebuznadores fueron alcaldes o regidores. Uno de los refranes recopilados por Correas reza, sólo que en forma afirmativa, lo mismo que el estandarte del pueblo del rebuzno: Rrebuznaron en balde el uno $i$ el otro alcalde (ibid., p. 5716 ).

5 "En cambio la legislación general, invariable, según parece, desde el siglo xv hasta el xvin -aunque soslayada por toda clase de fraudes- imponía a los merca- 
cúe al modelo evocado en La gilanilla: “. . dizen que somos como los jubones de los gavachos de Belmonte: rotos y grasientos y llenos de doblones". Pero resulta más sorprendente, y es revelador, que tal modelo se aplique también a los gitanos. Mirándolo bien, por ese hilo se saca un ovillo de cierta importancia: por poco que hable Cervantes de los gitanos, mencionará luego a los gabachos o hará que un morisco (poseedor de un tesoro secreto, como todos sus semejantes) se junte con unos franchotes que también ocultan su riqueza bajo sus esclavinas ${ }^{10}$.

Otros autores, Mateo Alemán por ejemplo, también utilizaron este tema del oro disimulado bajo harapos. Pero Alemán lo utiliza para denunciar los abusos de los mendigos fingidos. En Cervantes, en cambio, aparece exclusivamente asociado con esos "elementos foráneos" (gitanos, moriscos y gabachos) que destruyen, según él, la economía española. No por casualidad desfilan por el Coloquio representantes de estas tres castas ${ }^{11}$. También estas críticas han llegado a parecerle lugar común al lector de textos clásicos. Pero con ellas Cervantes aparece más próximo al pensamiento político de los arbitristas que a la tradición paremiológica ${ }^{12}$. Más exactamente, cosa en él constante, asocia elementos tomados de dicha tradición (la afición del peregrino extranjero por el vino) a otros propios de una crítica menos pintoresca $y$, por decirlo así, más "comprometida".

Esta diferencia es suficiente para probar que Cervantes se inspira en la tradición paremiológica y no lo contrario. Siempre es posible dudar, en efecto, de la anterioridad con respecto al Quijote de refranes recopilados hacia 1625, ya que la segunda parte de la obra es de 1615 . La hipótesis de que la gran popularidad del Quijole diera origen a una serie de refranes sobre los peregrinos extranjeros me parece insostenible por tres razones: 1) la ausencia de toda coincidencia formal entre los textos cervantinos y los refranes; 2) los pocos años transcurridos para que puedan aparecer tantas variantes; 3) por último, la carga crítica añadida que, en un momento dado, hace que se bifurque la sátira tra-

deres extranjeros la obligación de registrar a la llegada sus mercaderías, «y sacaı por el mismo puerto de entrada el precio que las vendieron, en otras mercaderías y no en oro ni en plata ni en moneda ". Batalllon, art. cit., p. 236.

10 "... juntéme con estos peregrinos, que tienen por costumbre de venir a España muchos dellos, cada año a visitar los santuarios della, que los tienen por sus Indias, y por certísima granjeria y conocida ganancia. Andanla casi toda, y no hay pueblo ninguno de donde no salgan comidos y bebidos, como suele decirse, y con un real, por lo menos, en dineros, y al cabo de su viaje, salen con más de cien escudos de sobra, que trocados en oro, o ya en el hueco de los bordones, o entre los remiendos de las esclavinas, o con la industria que ellos pueden, los sacan del reino y los pasan a sus tierras... Ahora es mi intención, Sancho, sacar el tesoro que dejé enterrado...", Quijote, ed, cit., t. 7, p. 332 .

11 Creo arbitraria, a pesar del gran interés de su trabajo, la separación introducida entre unos y otros por M. MoLHo, en su prólogo a la edición bilingüe de El casamiento engañoso y Coloquio de los perros, París, 1970.

12 Esta coincidencia con los arbitristas ya fue señalada por Amezúa en su edición crítica; mejor dicho, éste citó a Morel-Fatio a propósito de las críticas contra los moriscos ( $p_{4}$ 131). A pesar de su carácter explícito, nadie parece haber observado la relación establecida por Cervantes entre moriscos, gabachos y gitanos, que tam. bién figura en autores arbitristas. 
dicional. Cierto que, teóricamente, no es imposible el proceso inverso, que consiste en que un ataque se vacíe de sentido y pierda virulencia. Pero esto también necesitaría un plazo mucho más largo.

SEgunda SERIE de Refranes: El fRANCÉs CON SU JAMÓN.

Esta serie es menos rica. Conforme al estado actual de mis conocimientos, sólo consta de dos refranes. Por ser muy diferentes, bastan sin embargo para revelar la existencia de una tradición del francés aficionado al jamón, menos difundida que la del peregrino borracho, y que parece haber tenido más vigencia en el siglo xvı que en el xvn.

Estos proverbios, con comentarios que no permiten dudar de su carácter tradicional, confirmado además por su ritmo y musicalidad, figuran en dos obras cuyas fechas -1589 y 1605 - son capitales. Gracias a ellas, tenemos la prueba de que Cervantes aprovecha un doble filón folklórico al asociar la afición del peregrino por el vino al gusto por el jamón. He aquí los refranes anunciados:

1. "ANDRónico-Yo diré por él la mejor sentencia que él puede alegar, que al franchón no le quitéis el jambón.

Filaletes-No basta decir sentencias, si no son al propósito de lo que se dicen, y con alguna utilidad que dellas se siga..."13

2. “... que por eso se dijo el refrán El francés, hueso de tocino y la mesonera pan en el corpiño"14.

Puede ser que esta imagen del francés con su jamón -o con el hueso que roe hasta el fin, de acuerdo con su fama de avaricia- naciera en las regiones pirenaicas de España, más sensibilizadas que otras contra los vecinos. Así lo hace pensar, al menos, la forma dialectal, voluntariamente "gabacha" del refrán citado por el padre Pineda, escritor que vivió un tiempo en Aragón y muy hostil a los sacerdotes gascones ${ }^{15}$.

13 J. DE PINedA, Diálogos familiares de agricultura cristiana, BAE, t. 162, p. 179.

14 F. López de Úbeda, La picara Justina, en A. Valbuena Prat (ed.), La novela picaresca, Aguilar, 1956 , p. 739.

15 PinedA, op. cit., pp. 332, 163 y 327. No me parece casual que aparezca este refrán anti-gabacho en los Diálogos de un autor erasmista en los que figura un episodio que recuerda irresistiblemente la "armazón de caballería" de Don Quijote, como señaló recientemente Y. David-Peyre. Véase, por ejemplo, el siguiente párrafo: "Yo cursé primero bien en teología, y oponiéndome a beneficios nunca me dieron alguno y me moria de hambre; y por rcmediarme cursé otros tres años en medicina hasta graduarme de bachiller, y por no tener caudal para la costa del licenciamiento, quiso Dios que topé con un conde palatino tan hambriento como yo en la ventá de la Palomera, y convidéle a un lomo costil y a una bota de vino de Robleda de: Chavela, y allí me graduó de Licenciado, delante de los venteros y de los recuerdos, y tocaron la campana que tienen en la chimenea para llamar con ella a los descarriados en ticmpos de nieves, y después he ganado bien de comer". Citado por Y. DAvid Pryre, Le persomnage du médecin et la relation médecin malade dans la littérature iberique, xvie et xvije siécle [Tesis, Université de Lille III, p. 187]; otra edición: París, 1971. Por otra parte hay que tomar en cuenta que, según consta por los fichcros de la Real Academia, la forma franchón no se encuentra más que en el Padre Pineda. Debo la información a mi amigo Ignacio Soldevila. 
En mayor medida que las pullas contra los peregrinos borrachos, cargadas indudablemente de elementos críticos tocante a ortodoxia religiosa, esta imagen pertenece a una xenofobia primaria, ingenuamente impulsiva. Ahora bien: sabemos, por un pasaje del Quijote, que Cervantes sentía una gran curiosidad por las manifestaciones de esta xenofobia epidérmica, en virtud de la cual se satiriza a todo un grupo humano en razón de su alteridad. Mofa que da al grupo que la ejerce un sentido de superioridad, pero que surge, indudablemente, como una reacción defensiva. ¿Sería ese rechazo incomprensivo de todo "perspectivismo" lo que más llamó la atención de Cervantes? El caso es que puso en boca de Don Quijote, con intenciones satíricas no desprovistas de ambigüedad, un verdadero repertorio de apodos burlescos que podríamos llamar "topográficos", haciendo además que Don Quijote considerase normal la existencia de semejantes apodos entre vecinos ${ }^{16}$.

Acaso se aprecie mejor, precisamente comparándolo con la carga satírica de esas burlas "de luz y no de sangre", el alcance de la modificación imperceptible realizada por Cervantes al asociar dos tradiciones folklóricas hasta entonces separadas. La imagen del francés con su jamón, incluso con una posible alusión a la avaricia, es fundamentalmente una imagen caricaturesca. También domina la caricatura en la representación de un peregrino que, en jerga pintoresca, revela su devoción por el buen vino.

Al atribuir a todo extraniero que viaja por España el doble viático del jamón y el vino, Cervantes revigoriza considerablemente el valor crítico y da un sentido político a manifestaciones de una xenofobia casi ingenua.

En cierto sentido, ocurre algo similar cuando Cervantes agrega a la condición mágica tradicional - no ser hijo ni marido de una mujer infiel- la de no ser converso para poder contemplar el Retablo de las maravillas ${ }^{17}$. En este caso también, la condición mágica tradicional pierde su carácter de generalidad inocente y la obra puede aparecer como "fundada sobre la roca de los prejuicios de toda una época"18.

En el caso aquí examinado, la intervención de Cervantes es más sutil todavía, pues se contenta con asociar dos tradiciones preexistentes, que cobran un carácter explosivo por el mero hecho de verse asociadas.

16 "Siendo, pues, esto así, que uno solo no puede afrentar a reina, provincia, ni pueblo entero, queda en limpio que no hay para qué salir a la venganza del reto de la tal afrenta, pues no lo es; porque ;bueno sería que se matasen a cada paso los del pueblo de la Reloja con quien se lo llama, ni los cazoleros, berenjeneros, ballenatos, jaboneros, ni los otros nombres y apellidos que andan por ahi en boca de los muchachos y de gente de poco más o menos!...", Quijoțe, ed. cit., t. 6, pp. $185-186$.

17 Según Taylor, el motivo del embaucador que presenta un objeto mágico visible bajo ciertas condiciones no pertenece a la tradición folklórica sino a la literaria (véase D. Devoto, Introducción al estudio de don Juan Manuel y en particular de "El conde Lucanor", París, 1972; también sobre posibles antecedentes de El retablo de las maravillas el conocido estudio de M. Batallon, "Ulenspiegel y el Retablo de las maravillas de Cervantes" recogido en Varia lección de cldsicos españoles, Madrid, 1964, pp. 260-267).

$18 \mathrm{~J}$. Canavaggio, "Variations cervantines sur le thème du théâtré au thétre" RSH, 37 (1972), p. 67. 
Trátese de una verdadera adición (Retablo) o de una simple síntesis renovadora (vino + jamón), el fenómeno es el mismo. Lo tradicional se ve sometido a una reelaboración más o menos evidente, con lo que adquiere una virulencia que no tenía en su uso anterior. Proceso éste bien conocido por los folkloristas e investigadores que se dedican al estudio de la relación entre literatura culta y literatura popular ${ }^{19}$, y que debería ser tomado en cuenta para una revisión sistemática del papel desempeñado por el folklore en la obra de Cervantes.

Universitè de Lille III.

Monique Joly

\section{ASPECTOS TEMATICOS Y TÉCNICOS DE ROSITA DE VALLE-INCLÁN}

En las últimas décadas las obras de Ramón del Valle-Inclán han recibido un meticuloso escrutinio crítico. Sin embargo, una novelita ${ }^{1}$ ha escapado la atención de la mayoría ${ }^{2}$ de los estudiosos: Rosita (1903)3. A primera vista esta obra da la impresión de centrarse en un hecho sin trascendencia: el paseo por los jardines del Foreign Club de una pareja que no se ha visto por varios años. Si bien en Rosita lo que se describe es lo que sucede en este breve paseo, mucho de cuanto ocurre en este libro anticipa los escritos posteriores valleinclanescos. Por ello, la obra merece ser estudiada con detenimiento. Nuestro propósito, al hacerlo, es considerar sus temas y recursos técnicos, como lo indica el título de este trabajo.

\section{EL AMOR}

El amor aparece en esta novelita como una emoción falsa que se caracteriza por seguir varios estereotipos. No cabe duda de que la visión que se ofrece es crítica, y provoca en el lector una reacción similar a

I9 Cf. el estudio ejemplar de Marc Soriano, Les contes de Perrault, culture savante et traditions populaires, en que se analizan múltiples aspectos de ese proceso de reactivación del material folklórico.

1 Usamos el término "novelista" en el sentido de "pequeña novela". El propio Valle-Inclán consideró las obras que formaban Corte de amor como "novelas breves" en una nota suya a este volumen $\left(p_{4} 13\right)$. Citas y referencias a Rosita provienen de Corte de amor, Buenos Aires, 1942.

2 Ya Harold L. Boudreau se refirió al olvido de la critica de obras como Rosita: "The metamorphosis of the Ruedo ibérico" en Ramón del Valle-Inclan. An appraisal of his life and works. Ed. by A. N. Zahareas, R. Cardona and S. Greenfield, New York, 1968, p. 758].

3 Según Melchor Fernández Almagro (Vida y literatura de Valle-Inclán, Madrid, 1966, p. 88), Rosita apareció en 1903 como parte de Corte de amor y había sido rechazada en esa fecha por La Lectura. Datos similares, en lo que se refiere a la fecha de 1903, los ofrece José Rubia BArcia, "A synoptic view of Valle-Inclán's life and works", An appraisal, p. 11. Inexplicablemente, O. E. JACK ROBERTs (Definition and contrast of love in the "Corte de amor" and the "Sonatas" of Ramón del Valle. Inclan, tesis doctoral Louisiana State University, 1967, p. 16; también p. 70 notas 10 y 11) dice que Rosita formó parte de Femeninas en 1895. 